\title{
Gynecologic Oncology Board Certification
}

National Cancer Institute

\section{Source}

National Cancer Institute. Gynecologic Oncology Board Certification. NCI Thesaurus.

Code $C 17866$.

The process by which a person is tested and approved to practice in the specialty field of gynecologic oncology. 